\title{
Charged Particle Oscillations in Transient Plasmas Generated by Nanosecond Laser Ablation on Mg Target
}

\author{
Maricel Agop ${ }^{1,2}$, Ilarion Mihaila ${ }^{3}$, Florin Nedeff ${ }^{4}$ and Stefan Andrei Irimiciuc ${ }^{5, *(1)}$ \\ 1 Department of Physics, “Gh. Asachi” Technical University of Iasi, 700050 Iasi, Romania; m.agop@tuiasi.com \\ 2 Romanian Scientists Academy, 54 Splaiul Independentei, 050094 Bucharest, Romania \\ 3 Integrated Center for Studies in Environmental Science for North-East Region (CERNESIM), \\ Alexandru Ioan Cuza University of Iasi, 700506 Iasi, Romania; ilarion.mihaila@uaic.ro \\ 4 Mechanical Engineering, “Vasile Alecsandri” University of Bacau, Calea Mărăşeşti, 600115 Bacău, Romania; \\ florin_nedeff@ub.ro \\ 5 National Institute for Laser, Plasma and Radiation Physics, 409 Atomistilor Street, 077125 Bucharest, \\ Romania \\ * Correspondence: stefan.irimiciuc@inflpr.ro
}

Received: 12 January 2020; Accepted: 7 February 2020; Published: 17 February 2020

\begin{abstract}
The dynamics of a transient plasma generated by laser ablation on a $\mathrm{Mg}$ target was investigated by means of the Langmuir probe method and fractal analysis. The empirical data showcased the presence of an oscillatory behavior at short expansion times $(<1 \mu \mathrm{s})$ characterized by two oscillation frequencies and a classical behavior for longer evolution times. Space- and time-resolved analysis was implemented in order to determine main plasma parameters like the electron temperature, plasma potential, or charged particle density. In the motion fractal paradigm, a theoretical model was built for the description of laser-produced plasma dynamics expressed through fractal-type equations. The calibration of such dynamics was performed through a fractal-type tunneling effect for physical systems with spontaneous symmetry breaking. This allows both the self-structuring of laser-produced plasma in two structures based on its separation on different oscillation modes and the determination of some characteristics involved in the self-structuring process. The mutual conditionings between the two structures are given as joint invariant functions on the action of two isomorph groups of SL(2R) type through the Stoler-type transformation, explicitly given through amplitude self-modulation.
\end{abstract}

Keywords: laser ablation; charged particle oscillations; Langmuir probe; fractal analysis; Lie groups; joint invariant functions

\section{Introduction}

The fundamentals of laser-matter interactions have been at the core of developing a wide range of applications such as pulsed-laser deposition, material processing, or even medical applications. In the past few years, a great deal of interest has been given to fundamental aspects of high-power laser-matter interaction, especially in the context of the development of unique infrastructures that can generate novel applications. Pulsed-laser deposition has gained a lot of attention in the past 10 years as one of the best techniques to produce complex films with relatively complicated stoichiometry [1-3]. The technique has a proven flexibility in terms of the deposition geometry [4] and target or background gas nature $[5,6]$. Great advancements have been made toward understanding the fundamental aspects of laser ablation for a better control of the thin-film deposition technique. 
In the past years, the focus has also been on high-power laser interaction with materials, especially in the context of the development of a new generation of high-power lasers and their potential applicability in technological development and nuclear physics. Regardless of the application aims or the fluence regime used, there are several diagnostic techniques that can showcase the fundamental dynamics and behaviors of laser-produced plasmas. Over time, a wide range of investigation techniques have been developed like optical emission spectroscopy [7-9], mass spectrometry [10], or Langmuir probes [11-14] (LP), and these have been implemented to highlight phenomena like plasma structuring $[15,16]$, molecule formation $[17,18]$, elemental distribution in complex plasmas, and ejected particle behavior in various conditions. Out of all the techniques presented in the literature, LPs have shown great versatility being implemented for a wide range of materials $[11,19]$ and various irradiation conditions $[10,20]$. The LP method presents itself as a relatively simple diagnostic tool for plasma investigations, consisting of submerging a metallic electrode (of cylindrical, plane, or spherical geometry) in the plasma in order to record the ionic and electronic current or a mixture of the two currents, as selected by the applied voltage. The technique was first developed for steady-state discharge plasmas presenting local or global thermodynamic equilibrium. Laser-produced plasma (LPP) has a transient nature, with all its parameters presenting a complex spatial distribution and temporal dependence and being largely highly directional. The LP theory has been adapted for LPP by sampling the charged particle temporal traces and considering, besides the thermal movement, the drift movement of the ejected particle $[10,11]$. LP techniques offer insight into the ejected particle dynamics from a small plasma volume and, thus, can be implemented for axial and angular measurements, and it can easily be adapted for a wide range of diagnostics geometries. In the time-resolved approach to LP diagnostics, it can offer information from a wide set of plasma properties (electron temperature, plasma potential, charged particle density, collisions frequency, etc.) during the plasma expansion.

The flexibility of the technique allowed for some exciting findings. Charged particle oscillations were evidenced with the use of a single cylindrical probe [21], heated probe [12], and multiple probe $[22,23]$ configurations. Their nature is often debated with some reports presenting their roots in the dynamics of a plasma structure generated by electrostatic mechanisms [24], or induced by the transient double layers generated through plasma structuring [25]. Other novel theoretical approaches have been presented either in the framework of a fractal theoretical model [12,26] or built around Lorenz-type systems [27]. The complete behavior of plasma charged particles can be accounted for in the framework of the aforementioned fractal paradigm with the oscillatory behavior being explained through the presence of dissipative [12] or dispersive [26] effects, and the classical behavior is better showcased in the compact fractal hydrodynamic model [28]. The fractal paradigm proposes that every dynamic variable describing laser ablation plasma systems acts as the limit of families of functions. These functions can be differentiable for a non-zero scale resolution and non-differentiable for a null-scale resolution. The method is well-adapted for LPP dynamics, where the analyses conducted at a finite resolution scale imply the development of a new theoretical paradigm and new geometries. The motion curves can be seen as geodesics in the fractal space and forming a fractal fluid, where the ejected particles (the entities of the fluid) are replaced by their geodesics.

In this paper, we report on some novel results obtained by implementing the electrical diagnosis of laser ablation plasma produced on a Mg target. The Langmuir probe method was used to describe the behavior of laser-produced plasmas generated at different laser fluences and different measurement distances. A non-differentiable theoretical model based on a fractal interpretation of laser-produced plasma dynamics was used. The model is built around the interactions between the multiple plasma structures generated by laser ablation. In such contexts, the joint invariant functions on the action of two groups of SL(2R) type are given as mutual conditionings between the plasma substructures.

\section{Experimental Setup}

A schematic view of the experimental setup is presented in Figure 1. The experiments have been performed in a stainless-steel vacuum chamber pumped down to $2 \times 10^{-5}$ Torr residual pressure. 
The radiation from a Quantel Brilliant, Nd-YAG nanosecond laser (355 nm-3rd harmonic, pulse width $=5 \mathrm{~ns}, 10 \mathrm{~Hz}$ repetition frequency, variable fluences) was focused by a $\mathrm{f}=30 \mathrm{~cm}$ lens onto a magnesium target (the spot diameter at the impact point was approximately $0.3 \mathrm{~mm}$ ) placed in the vacuum chamber. The magnesium target rotated during the experiments and was electrically grounded from the vacuum chamber. Before each measurement, a surface-cleaning procedure was implemented in order to remove the oxide layer present at the surface of the target.

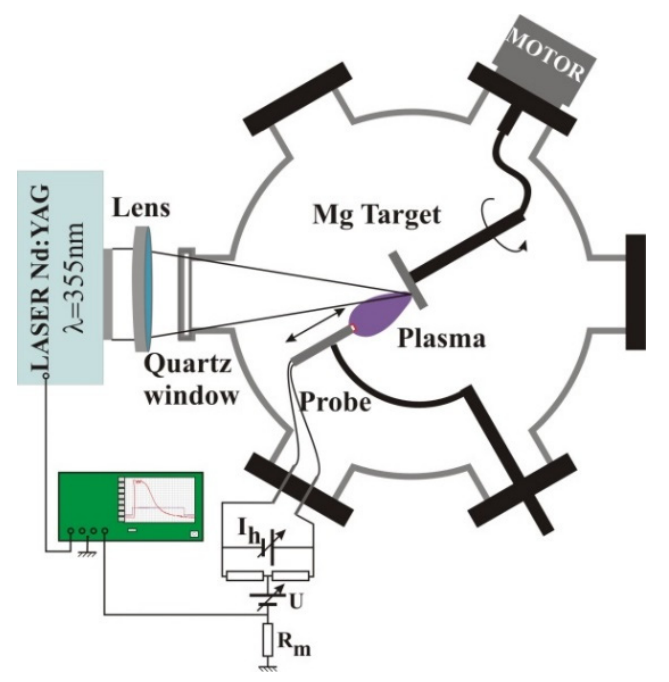

Figure 1. Experimental setup.

The ionic and electronic currents were collected from the ablation plasma plume using a tungsten-heated cylindrical Langmuir probe with a $0.25 \mathrm{~mm}$ diameter and $3 \mathrm{~mm}$ length. The probe was heated in order to avoid deposition of the target material on the probe, thus keeping constant the collecting area of the probe during measurements. In order to explore the dynamics of the expanding plasma, the probe was placed at different distances $(1.5,2,2.5$, and $3 \mathrm{~cm})$ with respect to the target, in the maximum expanding direction of the plume. The Langmuir probe was also biased by voltages on a range of values between -5 and $+10 \mathrm{~V}$ with a stabilized dc power source. Transitory signals were recorded by a digital oscilloscope and transferred to a PC for further analysis.

\section{Langmuir Probe Measurements}

For a bias of $\pm 5 \mathrm{~V}$ applied on the Langmuir Probe, we are able to collect the saturation ionic and electronic currents, displayed in Figure $2 \mathrm{a}, \mathrm{b}$, for two different fluences characterizing $2.45 \mathrm{~mm}^{3}$ plasma volume at $2 \mathrm{~cm}$ with respect to the target surface. By recording the saturation charge currents, we are assuring the collection of global ionic and electronic charge ejected as a result of the ablation process coupled with the ionization and neutralization process occurring during expansion of the laser-produced plasmas. We can distinguish different features for both saturation currents. The electronic current has a longer lifetime induced by the increased collision rate and the higher diffusion rate of the electrons as opposed to the ions. For the same applied voltage, the ionic $\mathrm{Mg}$ species have a lifetime of $3 \mu$ s while the electrons present almost twice as much, approximately $6 \mu \mathrm{s}$. There is, however, a recurring oscillating feature that can be found on all collected signals regardless of the applied voltage. The choice to represent here only the temporal trace for the saturation currents is supported by their higher amplitude and a better highlight of the smaller features, like the oscillatory part of the signal, noticeable below $1 \mu \mathrm{s}$. In the inset of Figure 2a,b we can see a zoomed in view of the oscillatory regime. We observe that for higher fluences, the first oscillatory maximum is reached at a shorter expansion time, with no significant increase in the amplitude of the current. The results indicate an increase in the kinetic energy of the ejected particle as induced by the increase in laser fluence, while the overall ejected 
charge remains quasi-constant. The oscillatory behavior has been previously reported for a wide range of materials, in classical or more complex ablation geometries [12,21-23].
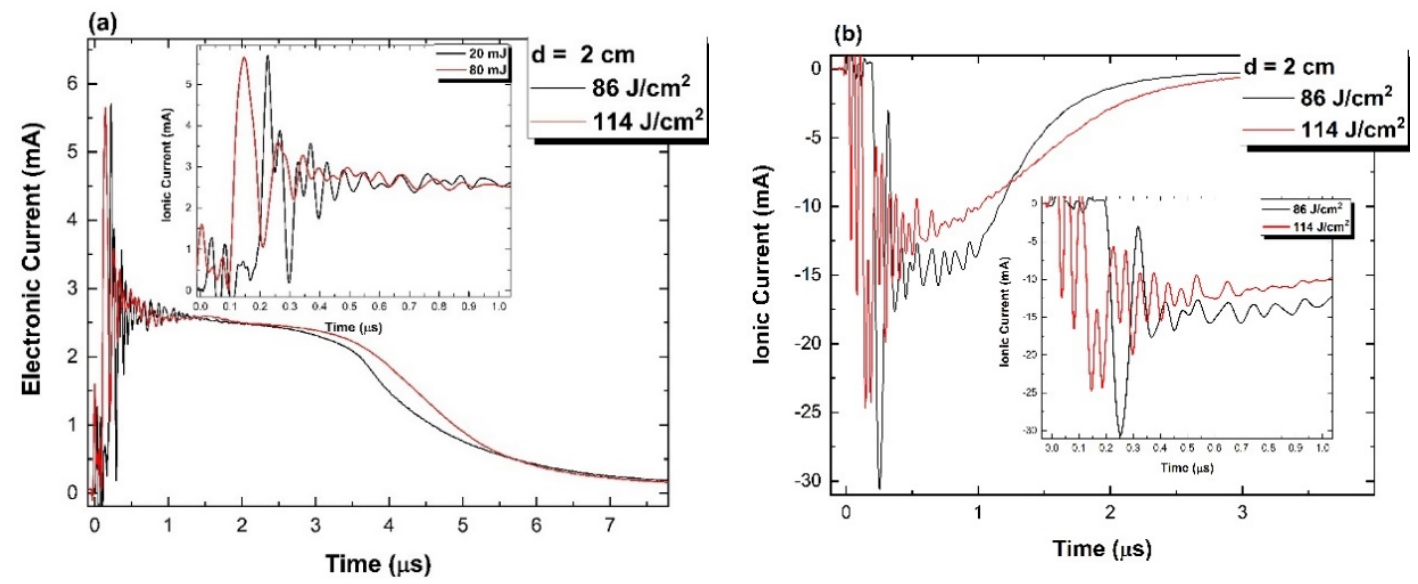

Figure 2. Temporal traces of the electronic (a) and ionic (b) current collected $2 \mathrm{~cm}$ from the target.

The nature of these oscillations is accepted, in some differentiable theoretical models, to be induced by the transient double layer forming as a result of the electrostatic ejection mechanism. This assertion would mean that the charged particles will oscillate with a frequency induced by the experimental conditions, as previously reported by our group in $[11,12,28]$. By performing fast Fourier transform (FFT) analysis on the collected signals, we observed two oscillation frequencies. These values showcase the strong dependence on the laser fluence (Figure 3a,b) and measurement distance (Figure 3c). The first oscillation frequency is of the order of tens of $\mathrm{MHz}$ (ranging from 15 up to $20 \mathrm{MHz}$ ), while the second one is of the order of a few $\mathrm{MHz}$ (ranging from 1 to $10 \mathrm{MHz}$ ). The results are in good agreement with our previous reports on Ni LPP, where we found values ranging from 5 up to $20 \mathrm{MHz}$ or with those reported on a wider range of metals reported in [25]. The second oscillation frequency is induced by the splitting of the laser-produced plasma during expansion into two plasma structures expanding with different velocities and the appearance of a secondary transient double layer, which will accelerate the slower plasma structure $[29,30]$. Each plasma structure is characterized by a unique oscillation frequency. With the increase in the laser fluence, we notice an increase in the oscillation frequency ( $5 \%-50 \%$ depending on the measurement distance), which indicates the generation of a stronger electrical field generated through Coulomb explosion, followed by saturation for fluences higher than $80 \mathrm{~J} / \mathrm{cm}^{2}$. The observed oscillations are damped after approximately $1 \mu \mathrm{s}$. This can also be seen from the oscillation frequency evolution with the measurement distance where we notice a decrease of about $25 \%$ for both measured frequencies. Admittedly, the laser fluence values are much higher than those used in the pulsed-laser deposition or material processing applications, where we can find reports of fluences below $5 \mathrm{~J} / \mathrm{cm}^{2}$ [1-6], depending on the irradiation conditions and the nature of the thin film envisioned in each report. However, the results become relevant for the fundamental new generation of high-power laser-matter interactions.

The Langmuir probe theory is reportedly limited only for longer expansion times where the theoretical assumptions are met. For transient plasma and laser-produced plasma in general, the temporal evolution of some of the parameters $\left(T_{e}, V_{p}, n_{i}\right)$ is determined by evaluating all the recorded I-V characteristics, in the hypothesis that in the moments of time selected for analysis, the plasma has properties imposed by LP theory. This hypothesis cannot always be verified, as the implementation of the technique induces some limitations. In the incipient part of the evolution $(<1 \mu \mathrm{s})$ and in the proximity of the target (few $\mathrm{mm}$ ), the probe-collecting surface is significantly larger than the measured plasma volume; therefore, LP theory is no longer valid, as the measurement electrode must not impact the plasma around it. In these special space-time coordinates, data reveals the presence of 
a complex oscillatory regime of the ionic and electronic temporal traces, which are also part of our reported results in this paper [11].
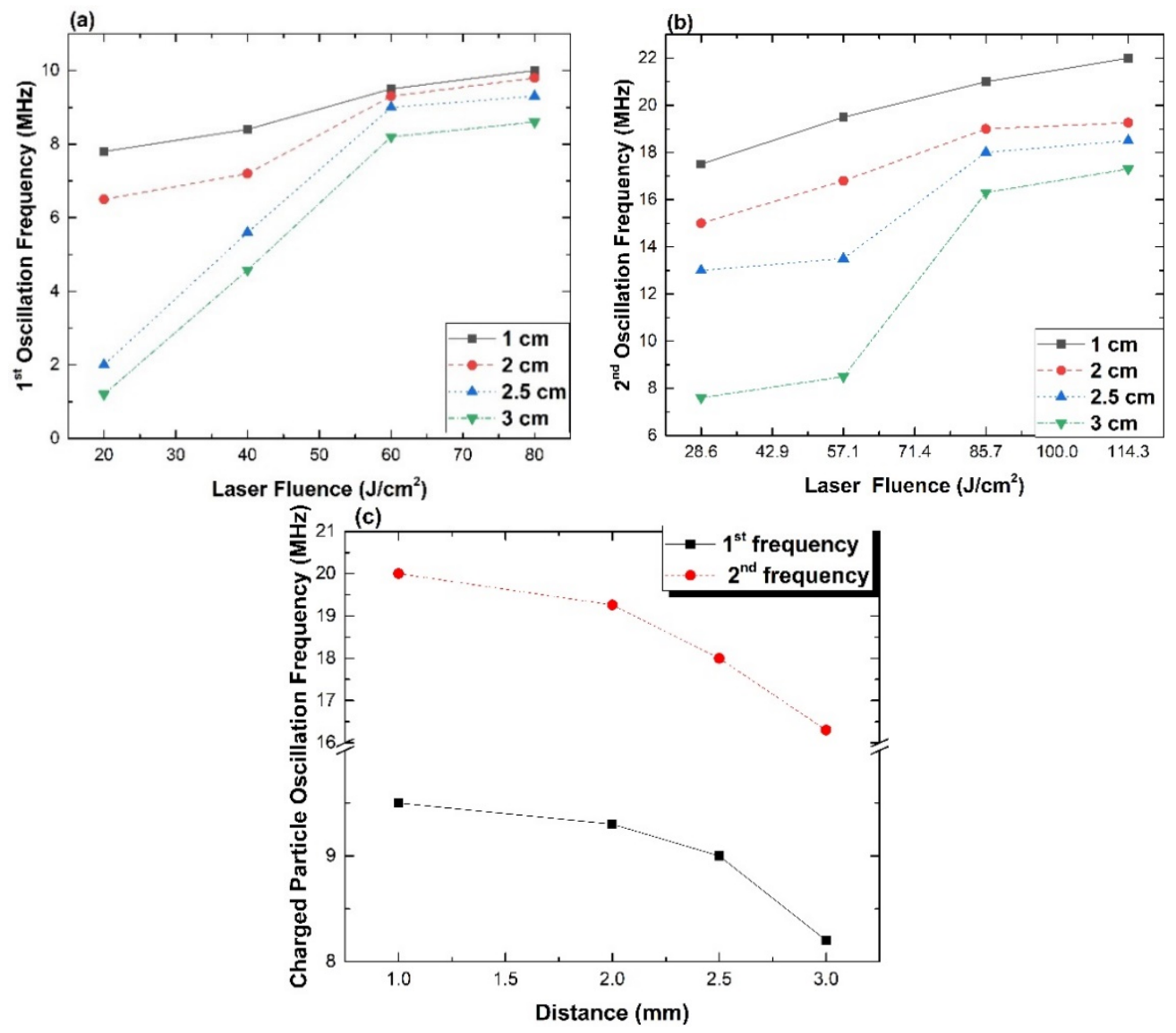

Figure 3. Oscillation frequency evolution with laser fluence $(\mathbf{a}, \mathbf{b})$ and measurement distance (c).

This allows us to characterize the temporal evolution of some plasma parameters like electron temperature, plasma potential, or ionic density. In order to achieve that, we implemented the methodology used in [11]. Briefly, consecutive bias potentials (in 50 points) ranging from -5 to $+10 \mathrm{~V}$ were applied on the heated probe, and the plasma currents were collected from a well-defined plasma volume. The probe was moved at various distances with respect to the target's surface. An example is given in Figure 4. As anticipated in the previous paragraphs, all signals follow a similar pattern with an oscillatory part for short evolution times followed by a classical decreasing trend.

For a time-span of $6 \mu$ s using a step of $1 \mu \mathrm{s}$, we reconstruct the I-V characteristics for each moment in time. In Figure 5, we have presented representative characteristics describing the LPP at various distances after $1 \mu \mathrm{s}$ (Figure 5a) and, for a fixed distance of $2.5 \mathrm{~cm}$, the reconstructed characteristics at different moments in time (Figure $5 b$ ). We notice that for an instant temporal sequence, the axial dependence of the I-V characteristics is not significant; however, there is a substantial shift toward positive floating potential. This is due to the spatial distribution of the two sets of charges during expansion. The electrons generally have a more uniform distribution in the plasma; however, in light of the electrostatic ejection mechanism, the first electrons ejected will spatially occupy the position in front of the ejected ions. Although during expansion, there are other phenomena that need to be considered like ion neutralization, secondary ionizations, or molecule formation [18], this shift in the floating potential showcases the change in the ion-to-electron ratio within the plasma volume for a fixed moment in time $(1 \mu \mathrm{s})$. The spatiotemporal evolution of all main plasma parameters considered here (electron temperature and plasma potential) follow a classical quasi-exponential decrease. To determine the aforementioned plasma parameters, we treated the I-V characteristics following the procedure 
from $[10,11]$. Briefly, by applying a logarithm procedure to the I-V characteristic curve, we will obtain a distribution that is defined by a linear increase, an inflection point, and a saturation region. The slope of that linear increase will define the electron temperature while the inflection point will be the plasma potential (Figure 5e). Details on this procedure can also be seen in [10-12].

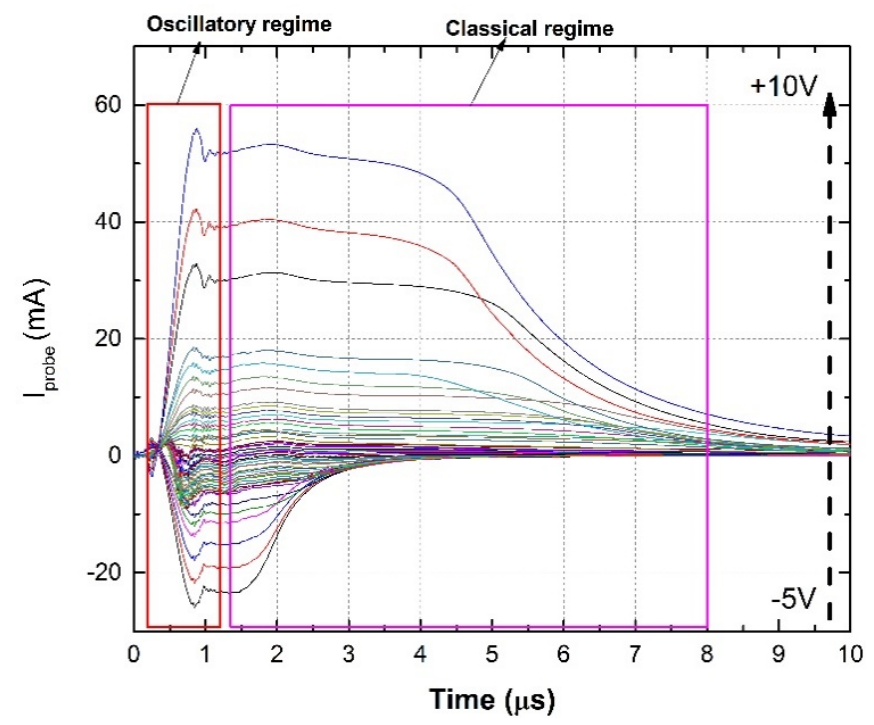

Figure 4. Temporal traces of the ionic and electronic currents recorded for a laser fluence of $56 \mathrm{~J} / \mathrm{cm}^{2}$ at $2.5 \mathrm{~cm}$ from the target.

The increase in the laser fluence leads to higher electron temperatures and plasma potentials (Figure $5 c, d$ ). The highest values found here are $T_{e}=1 \mathrm{eV}$ and $V_{p}=9.3 \mathrm{~V}$ for the measurements performed at $1.5 \mathrm{~cm}$ and a laser fluence of approximately $114 \mathrm{~J} / \mathrm{cm}^{2}$, while the lowest are found for $28 \mathrm{~J} / \mathrm{cm}^{2}$ with the values decreasing with almost one order of magnitude: $T_{e}=0.1 \mathrm{eV}$ and $\mathrm{V}_{\mathrm{p}}=2 \mathrm{~V}$. For a fixed distance, the time-resolved analysis reveals important changes in both shapes of the I-V characteristics. These changes are induced by the decrease in all the plasma parameters as indicators of the laser-produced plasma expansion, particle density, and particle energy losses, showcased in the inset of Figure $5 b$ where the temporal evolutions of $T_{e}$ and $V_{p}$ are presented.

The influence of the laser fluence over some plasma parameters is generally known. A higher laser fluence usually leads to the ejection of a higher density of particles with a higher kinetic and thermal energy. For our conditions, we synthesized the data in Figure 6a where we present the evolution of the ionic density with the laser fluence at various distances, and in Figure 6b, where the evolution of the ion drift velocity is presented. The results are in line with other reports where the same increase followed by a saturation regime can be seen. The ion drift velocities were determined by plotting the evolution of the ionic current maxima as a function of space and time. The slope of that representation defines the drift velocity.

The overall effect of the laser fluence depicted in Figures 3 and 6 is to enhance the oscillatory movement of the charged particles, increase the current densities, and, overall, increase the kinetic movement of the plasma. The kinetic enhancement of the LPP reaches a saturation regime, however, where neither the particle density nor expansion velocities increase. This could mean that the energy is lost on expelling large structures (clusters and nanoparticles). The result is in line with our previous results reported in [12] where the presence of a third plasma structure was seen in the particle velocity distribution at high laser fluence. The interaction between the two plasma structures and their unique signatures in the ionic and electronic saturation currents is still to be understood. The subtle difference in plasma plume dynamics at high fluence as opposed to the usual lower values used in applications such as PLD still needs to be investigated by other experimental techniques and cemented through comprehensive theoretical modeling. 

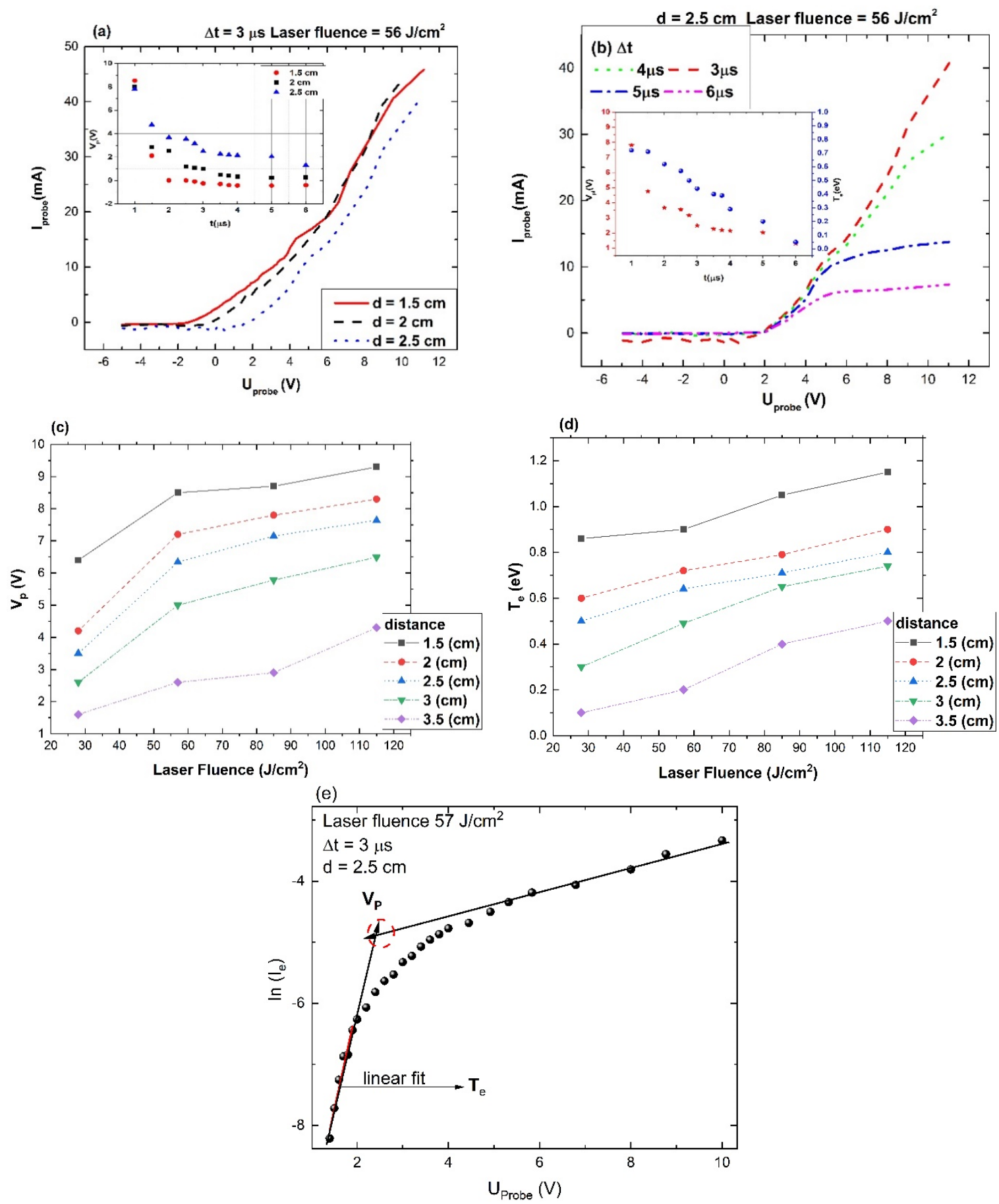

Figure 5. Evolution of the I-V characteristics in time (a) and space (b); plasma potential (c) and electron temperature (d) evolution with distance and laser fluence and an example of the logarithmic representation of the $\mathrm{I}-\mathrm{V}$ characteristic $(\mathbf{e})$. 

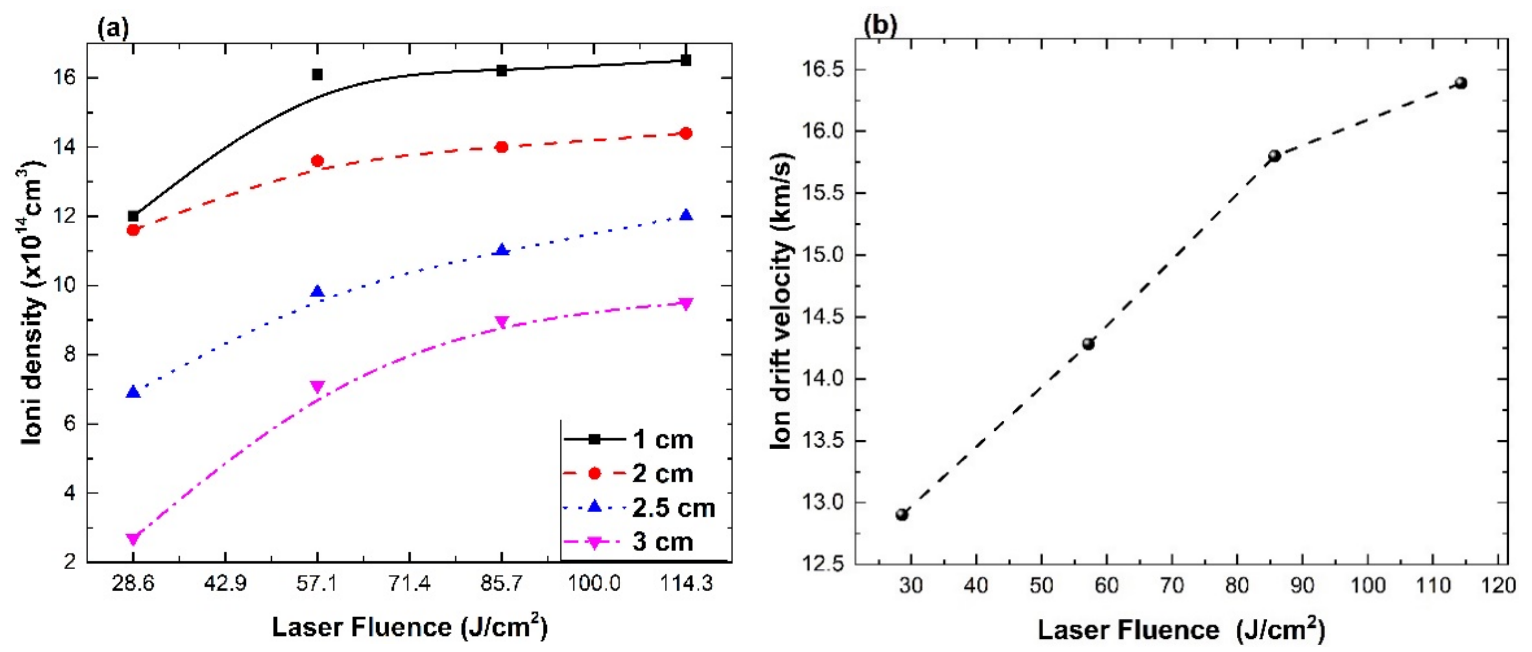

Figure 6. Ionic density (a) and velocity (b) evolution with the laser fluence.

\section{Mathematical Model}

\subsection{Ablation Plasma as a Fractal Medium}

Ablation plasma behaves like a fractal medium induced by the collisions process between its entities (electrons, ions, neutrals), with the results of the interactions taking the form of excitations, ionizations, or recombination $[12,26,27]$. Such an assumption can be theoretically sustained by this typical example: Between two successive collisions, the trajectory of the plasma particles is a straight line that becomes non-differentiable at the impact point. Considering now that all the collision impact points form an uncountable set of points, the trajectories of plasma particles become continuous but nondifferentiable curves, i.e., a fractal. Some follow-up clarifications from a mathematical and physical point of view need to be made: (i) If the laser-produced plasma expands at a lower background pressure (a lack of collisions between the ejected particles), it mimics a pre-fractal and approximates a fractal-sparse set [31]; (ii) if the ablation plasma expands at higher background pressures (an expansion rich in collisions), it mimics a fractal-dense set.

In such a context, fractal theories of motion [31-34] become functional for describing the dynamics of ablation plasma. Significant advancements for the implementation of a fractal paradigm to a classical physical system has been done in a series of papers [35-37], especially for the problem of the Schrodinger representation of fractal type [38-41]. The fundamental hypothesis of our model is that the dynamics of all ablation plasma entities can be characterized by fractal motion curves. These continuous but non-differentiable curves display the property of self-similarity at every point, which can be translated into a property of holography (every part reflects the whole). We can further use "holographic implementations" of the dynamics of any ablation plasma entity, for example, through special fractal-type fractal "regimes" (i.e., describing dynamics by using fractal-type equations at various scale resolutions).

The merit of developing theoretical models based on a fractal paradigm has been shown consistently by our group in the past 12 years. Our approach comes as an alternative to classical approaches in an attempt to offer a general model that can be adapted for various specific processes that need to be investigated. The model is complex and uses non-differentiable mathematics and is strongly linked to concepts from fractal physics. However, classical approaches to describe laser-produced plasma dynamics present some limitations. Theoretical models used to describe the laser ablation plasmas define either the first moments of LPP formation and one-dimensional expansion that occurs for small distances and short evolution times (dominated by laser-target interactions and fundamental particle removal mechanisms), or the later stages of expansion (a few $\mu \mathrm{m}$ after the laser pulse, starting from a few $\mathrm{mm}$ from the target), where the LPP has a three-dimensional expansion that is often described 
using classical hydrodynamics. Due to these major discrepancies in the space-time interaction and expansion scales, significant complications arise in developing a unique model that can cover all the complex phenomena involved in laser ablation. One of the domains of theoretical physics that has, at its core, the scale dependences of dynamic parameters is scale relativity theory (SRT) [31-34]. In SRT, the complete set of equations required to describe the dynamics of certain systems needs to contain the movement equations and those related to the non-differentiable processes. The non-differentiability indicates that for a plasma medium, the plasma entity evolution is defined by fractal curves (moving in a straight line between two collisions, and becoming nondifferentiable at the impact point) with the geodesics of fractal entities replacing the dynamics of plasma entities. In such a context, we can approximate the ablation plasma plume dynamics with that of a fractal fluid without interactions. The fractal hydrodynamic model [23] has been used in our group in recent years to describe the self-structuring of the ablation cloud during expansion and the evolution of the plasma parameters [27].

With respect to connections between the plasma parameters and those defined by the model, over time, we reported some clear relations. In [28], we give direct relations between the electron temperature and the fractal potential, ion density, normalized fractal entities density, electron thermal velocity, and non-differentiable velocity, and between the Debye length and the normalized specific length. In [11], we study the relationship between the fractalization degree given in the model and the heterogeneity of the ablated cloud in terms of particle sizes and properties.

\subsection{Scale Covariant Derivative and Geodesics Equations}

Let us now consider the scale covariance principle (the physical laws applied to the dynamics of the laser-produced plasmas are invariant with respect to scale resolution transformations [31-34]) and postulate that the transition from standard (differentiable) plasma physics to fractal (non-differentiable) plasma physics can be implemented by replacing the standard time derivative $d / d t$ by the non-differentiable operator $\hat{d} / d t[35-37]$ :

$$
\frac{\hat{d}}{d t}=\partial_{t}+\hat{V}^{l} \partial_{l}+\frac{1}{4}(d t)^{\left(\frac{2}{D_{F}}\right)-1} D^{l p} \partial_{l} \partial_{p}
$$

where

$$
\begin{gathered}
\hat{V}^{l}=V_{D}^{l}-V_{F^{\prime}}^{l}, \\
D^{l p}=d^{l p}-i \overline{\bar{d}}, \\
d^{l p}=\lambda_{+}^{l} \lambda_{+}^{p}-\lambda_{-}^{l} \lambda_{--}^{p} \\
\bar{d}^{l p}=\lambda_{+}^{l} \lambda_{+}^{p}+\lambda_{-}^{l} \lambda_{-\prime}^{p} \\
\partial_{t}=\frac{\partial}{\partial t^{\prime}}, \partial_{l}=\frac{\partial}{\partial X^{l}}, \partial_{l} \partial_{p}=\frac{\partial}{\partial X^{l}} \frac{\partial}{\partial X^{p}}, i=\sqrt{-1}, l, p=1,2,3 .
\end{gathered}
$$

In the above relations, $\hat{V}^{l}$ is the complex velocity, $V_{D}^{l}$ is the differentiable velocity independent of the scale resolution $d t, V_{F}^{l}$ is the nondifferentiable velocity dependent on the scale resolution, $X^{l}$ is the fractal spatial coordinate, $t$ is the non-fractal time with the role of an affine parameter of the motion curves, $D^{l p}$ is the constant tensor associated with the differentiable-non-differentiable transition, $\lambda_{+}^{l}$ is the constant vector associated with the forward differentiable-nondifferentiable physical processes, $\lambda_{-}^{l}$ is the constant vector associated with the backward differentiable-nondifferentiable physical processes, and $D_{F}$ the fractal dimension of the movement curve. For the fractal dimension, we can choose any definition, for example, the Kolmogorov-type fractal dimension or Hausdorff-Besikovici-type fractal dimension [42]. However, once chosen and becoming operational, it needs to be constant and arbitrary: $D_{F}<2$ for the correlative physical processes; $D_{F}>2$ for the non-correlative physical processes [31-34].

Now, the non-differentiable operator plays the role of the scale covariant derivative; namely, it is used to write the fundamental equations of ablation plasma dynamics in the same form as in the classic (differentiable) case. Under these conditions, accepting the functionality of the scale covariant principle, i.e., applying the scale covariant derivative, Equation (1), to the complex velocity field, Equation (2), 
in the absence of any external constraint, the geodesics equation of the ablation plasma takes the following form [32-34]:

$$
\frac{\hat{d} \hat{V}^{i}}{d t}=\partial_{t} \hat{V}^{i}+\hat{V}^{l} \partial_{l} \hat{V}^{i}+\frac{1}{4}(d t)^{\left(\frac{2}{D_{F}}\right)-1} D^{l k} \partial_{l} \partial_{k} \hat{V}^{i}=0
$$

This means that the fractal local acceleration $\partial_{t} \hat{V}^{i}$, the fractal convection $\hat{V}^{l} \partial_{l} \hat{V}^{i}$, and the fractal dissipation $D^{l k} \partial_{l} \partial_{k} \hat{V}^{i}$ of any ablation plasma entity balance themselves at any point of the motion fractal curve. Moreover, the presence of the complex coefficient of viscosity type $4^{-1}(d t)^{\left(\frac{2}{D_{F}}\right)-1} D^{l k}$ in the laser-produced plasma (LPP) dynamics specifies that it is a rheological medium. Therefore, the plasma structures have memory, as a datum, by their own structure.

If the fractalization in the dynamics of LPP is achieved by Markov-type stochastic processes, which involve Lévy-type movements [31,42] of the plasma entities,

$$
\lambda_{+}^{i} \lambda_{+}^{l}=\lambda_{-}^{i} \lambda_{-}^{l}=2 \lambda \delta^{i l},
$$

where $\lambda$ is a coefficient associated with the differentiable-nondifferentiable transition and $\delta^{i l}$ is Kronecker's pseudo-tensor.

Under these conditions, the geodesics equation takes the following simple form:

$$
\frac{\hat{d} \hat{V}^{i}}{d t}=\partial_{t} \hat{V}^{i}+\hat{V}^{l} \partial_{l} \hat{V}^{i}-i \lambda(d t)^{\left(\frac{2}{D_{F}}\right)-1} \partial^{l} \partial_{l} \hat{V}^{i}=0 .
$$

For irrotational motions of the ablation plasma entities, the complex velocity field $\hat{V}^{i}$ takes the following form:

$$
\hat{V}^{i}=-2 i \lambda(d t)^{\left(\frac{2}{D_{F}}\right)-1} \partial^{i} \ln \Psi .
$$

Then, substituting Equation (6) in Equation (5), the geodesics equation (Equation (5)) (for details, see method from [42-44]) becomes a Schrödinger-type equation at various scale resolutions:

$$
\lambda^{2}(d t)^{\left(\frac{4}{D_{F}}\right)-2} \partial^{l} \partial_{l} \Psi+i \lambda(d t)^{\left(\frac{2}{D_{F}}\right)-1} \partial_{t} \Psi=0 .
$$

The variable $\Phi=-2 i \lambda(d t)^{\left(2 / D_{F}\right)-1} \ln \Psi$ defines, through Equation (6), the complex scalar potential of the complex velocity field, while $\Psi$ corresponds to the ablation plasma state of fractal type. Both variables, $\Phi$ and $\Psi$, have no direct physical meaning, but possible "combinations" of them can acquire it if they satisfy certain conservation laws.

Let us make explicit such a situation for $\Psi$. For this purpose, we first notice that the complex conjugate of $\Psi$, that is, $\bar{\Psi}$, satisfies through Equation (7) the following equation:

$$
\lambda^{2}(d t)^{\left(\frac{4}{D_{F}}\right)-2} \partial^{l} \partial_{l} \bar{\Psi}-i \lambda(d t)^{\left(\frac{2}{D_{F}}\right)-1} \partial_{t} \bar{\Psi}=0 .
$$

Multiplying Equation (7) by $\bar{\Psi}$ and Equation (8) by $\Psi$, subtracting the results, and introducing the notations,

$$
\rho=\Psi \bar{\Psi}, \quad J=i \lambda(d t)^{\left(2 / D_{F}\right)-1}(\Psi \nabla \bar{\Psi}-\bar{\Psi} \nabla \Psi),
$$

we can obtain the conservation law of the states density of fractal type:

$$
\partial_{t} \rho+\nabla J=0
$$

In Equation (10), $\rho$ corresponds to the states density of fractal type and $J$ corresponds to the states density current of fractal type. 


\subsection{Ablation Plasma Behavior through a Special Tunneling Effect of Fractal Type}

In the following, we will perform an application of the mathematical model previously mentioned by analyzing one-dimensional (1-D) stationary dynamics in physical systems with spontaneous symmetry breaking in the form of a tunneling effect of fractal type. The results will be corelated/calibrated with the dynamics of an LPP from the perspective of generating two plasma structures, as well from the perspective of some essential characteristics of these structures.

Let us consider the following equation of fractal type [32-34]:

$$
\lambda^{2}(d t)^{\left(\frac{4}{D_{F}}\right)-2} \partial^{l} \partial_{l} \Psi+i \lambda(d t)^{\left(\frac{2}{D_{F}}\right)-1} \partial_{t} \Psi-\frac{U}{2} \Psi=0,
$$

which has an external restriction in the form of the scalar potential $U$. For the moment, the scalar potential is not explicitly defined.

In the 1-D stationary case, Equation (11) becomes

$$
\lambda^{2}(d t)^{\left(\frac{4}{D_{F}}\right)-2} \partial_{z z} \Psi(z, t)+i \lambda(d t)^{\left(\frac{2}{D_{F}}\right)-1} \partial_{t} \Psi(z, t)-\frac{U}{2} \Psi(z, t)=0 .
$$

If the scalar potential $U$ is time-independent, $\partial_{t} U=0$, Equation (12) admits the stationary solution (for details on the method, see [42-44]):

$$
\psi(z, t)=\theta(z) \exp \left[-\frac{i}{2 m_{0} \lambda(d t)^{\left(\frac{2}{D_{F}}\right)-1}} E t\right],
$$

where $E$ is the fractal energy of the plasma entity, $\theta(x)$ is the stationary state of fractal type of the plasma entity, and $m_{0}$ is the fractal rest mass of the plasma entity. Then, $\theta(x)$ becomes a solution of the fractal non-temporal equation:

$$
\partial_{z z} \theta(z)+\frac{1}{2 m_{0} \lambda^{2}(d t)^{\left(\frac{4}{D_{F}}\right)-2}}(E-U) \theta(z)=0 .
$$

In the following, we will assume that the behavior of the LPP, such as self-structuring in two different substructures (Coulomb and thermal) based on the plasma plume separation on different oscillations modes, can be mimicked by spontaneous symmetry breaking. The mathematical procedure will imply the use of Equation (14) for a scalar potential $U$ characteristic to the spontaneous symmetry breaking (see Figure 7). From the theory of spontaneous symmetry-breaking systems [31,42], the shape of the effective potential is already well-known and has the following expression:

$$
V_{e f}(z)=V_{0}\left[\left(\frac{z}{z_{0}}\right)-1\right]^{2}, V_{0}=\text { const, } z_{0}=\text { const. }
$$

The exact integral of the stationary Schrödinger equation of fractal type,

$$
\partial_{z z} \theta(z)+\frac{1}{2 m_{0} \lambda^{2}(d t)^{\left(\frac{4}{D_{F}}\right)-2}}\left(E-V_{0}\left[\left(\frac{z}{z_{0}}\right)-1\right]^{2}\right)=0,
$$

is not achievable. The determination of the eigenfunctions and eigenvalues $\{\theta, E\}$ can be attained only by successive approximations, order by order, in the framework of stationary perturbation theory, starting from the 0 order, from the following eigenfunctions and eigenvalues:

$$
\left\{\varphi_{n}(z), E_{n}=2 m_{0} \lambda(d t)^{\left(2 / D_{F}\right)-1}\left(n+\frac{1}{2}\right)\right\}_{n \in N^{\prime}}
$$


of the fractal harmonic linear oscillator. To avoid this setback, a simplifying hypothesis can be introduced considering some approximations, as the equation cannot be integrated in a closed form. The following statements can be made:

(i) At $|z|=2\left|z_{0}\right|, V_{e f}$ is much larger than $V_{0}$, which means to the right of $z_{0}$, and to the left of $-z_{0}$, the increase in the effective potential is fast enough so that it can be approximated with two infinite vertical walls at $z \pm l$, with $l \geq z_{0}$ (mandatory);

(ii) Because $\mathrm{E}_{0}$ is placed below half of the local maxima $V_{0}$ and, at $|z|=0.5\left|z_{0}\right|, V_{\text {ef }}$ is already higher than $V_{0} / 2$, the same thought process can done in $z \pm d$, with $d<z_{0}$ (mandatory), by placing in each of these points a vertical wall of $V_{0}$ height. Therefore, $V(\infty)$ for $U \equiv V(z)$ becomes a condition for the spontaneous symmetry breaking, as well as for $U^{\prime}$ 's symmetry.

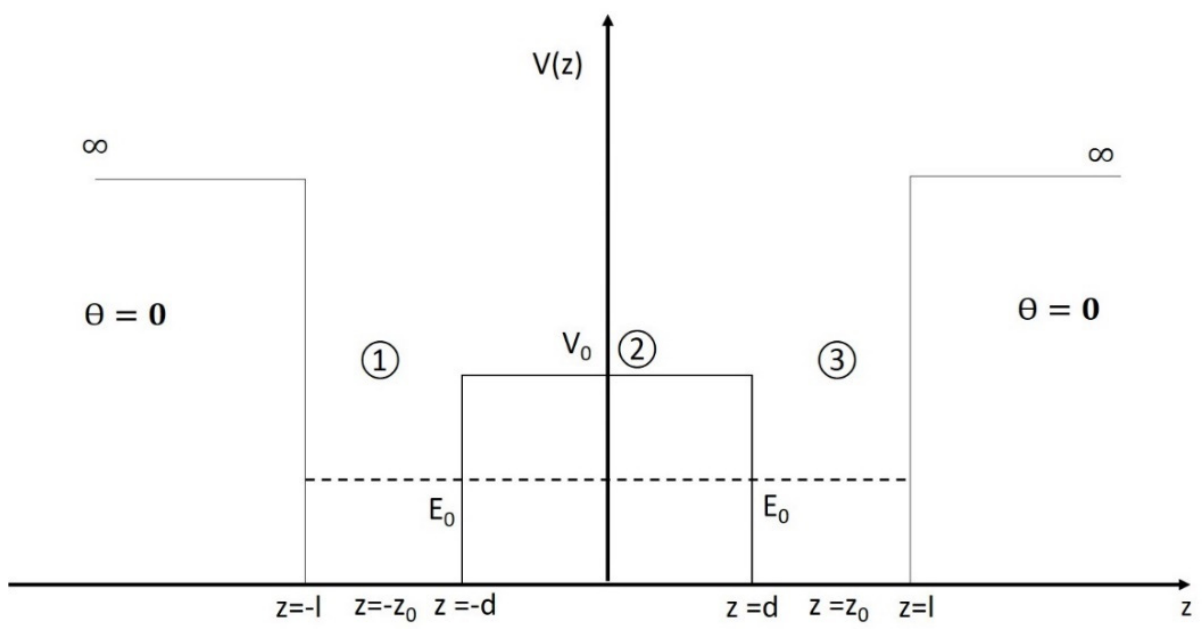

Figure 7. The effective potential in the case of the tunneling effect of fractal type for a physical system with spontaneous symmetry breaking (where $E_{0}$ is the fundamental energy level, $V_{0}$ is the height of the potential barrier, $z=2 d$ is the width of the potential barrier, and $z=l-d$ is the width of the potential well).

In these conditions, Equation (14) takes the following form:

$$
\frac{d^{2} \theta_{\alpha}}{d z^{2}}+\frac{1}{2 m_{0} \lambda^{2}(d t)^{\left(\frac{4}{D_{F}}\right)-2}}\left[E-V_{\alpha}\right] \theta_{\alpha}=0, \alpha=\overline{1,3}
$$

For each of the three regions, the solutions of Equation (15) are

$$
\begin{aligned}
& \theta_{1}(z)=C_{+} e^{i k z}+C_{-} e^{-i k z} \\
& \theta_{2}(z)=B e^{q z}+C e^{-q z} \\
& \theta_{3}(z)=D_{+} e^{i k z}+D_{-} e^{-i k z}
\end{aligned}
$$

with

$$
\begin{aligned}
& k=\left[\frac{E}{2 m_{0} \lambda^{2}(d t)^{\left(\frac{4}{D_{F}}\right)-2}}\right]^{\frac{1}{2}}, \\
& q=\left[\frac{V_{0}-E}{2 m_{0} \lambda^{2}(d t)^{\left(\frac{4}{D_{F}}\right)-2}}\right]^{\frac{1}{2}},
\end{aligned}
$$

and $C_{+}, C_{-}, B, C, D_{+}, D_{-}$are integration constants. 
Due to the infinite potential in the two extreme regions, $|z|>l$, the state function of fractal type, $z= \pm l$, implies

$$
\begin{gathered}
\theta_{2}(-l)=C_{+} e^{-i k l}+C_{-} e^{i k l}=0, \\
\theta_{3}(l)=D_{+} e^{i k l}+D_{-} e^{-i k l}=0 .
\end{gathered}
$$

As the states density of fractal type, $|\psi|^{2}$, is not altered by the multiplication of the state function of fractal type in the form of a constant phase factor of fractal type, the two equations for $C_{ \pm}$and $D_{ \pm}$ can be immediately solved by imposing the forms,

$$
\begin{aligned}
& C_{+}=\frac{A}{2 i} e^{i k l}, C_{-}=-\frac{A}{2 i} e^{-i k l}, \\
& D_{+}=\frac{D}{2 i} e^{-i k l}, D_{-}=-\frac{D}{2 i} e^{i k l},
\end{aligned}
$$

so that $\theta_{1,3}$ are given through simple expressions:

$$
\begin{aligned}
& \theta_{1}(z)=A \sin [k(z+l)], \\
& \theta_{3}(z)=D \sin [k(z-l)] .
\end{aligned}
$$

These, along with $\psi_{2}$, lead to the concrete form of "alignment conditions" in $z= \pm d$ :

$$
\begin{gathered}
\theta_{1}(-d)=\theta_{2}(-d), \theta_{2}(d)=\theta_{3}(d), \\
\frac{d \theta_{1}}{d z}(-d)=\frac{d \theta_{2}}{d z}(-d), \frac{d \theta_{2}}{d z}(d)=\frac{d \theta_{3}}{d z}(d),
\end{gathered}
$$

namely,

$$
\begin{aligned}
& e^{-q d} B+e^{q d} C=A \sin [k(l-d)], \\
& q e^{-q d} B-q e^{q d} C=k A \cos [k(l-d)] \text { in } z=-d, \\
& e^{q d} B+e^{-q d} C=-D \sin [k(l-d)], \\
& q e^{q d} B-q e^{-q d} C=k D \cos [k(l-d)] \text { in } z=d .
\end{aligned}
$$

Due to the algebraic form of the two equation pairs, in order to establish the concrete expression of the "secular equation" (for eigenvalues $E$ of the energy), $\Delta[E]=0$, we avoid calculating the 4th order determinant, $\Delta[k(E), q(E)]$, formed with the amplitude coefficients of fractal type, $A, B, C, D$, by employing the following: We note, with $\rho$, the ratio $C / B$, and we divide the first equation with the second one for each pair. It results in

$$
\begin{aligned}
& \frac{e^{2 q d} \rho+1}{e^{2 q d} \rho-1}=-\frac{q}{k} \operatorname{tg}[k(l-d)], \\
& \frac{e^{-2 q d} \rho+1}{e^{-2 q d} \rho-1}=\frac{q}{k} \operatorname{tg}[k(l-d)],
\end{aligned}
$$

which leads to the equation for $\rho$ :

$$
\frac{e^{2 q d} \rho+1}{e^{2 q d} \rho-1}+\frac{e^{-2 q d} \rho+1}{e^{-2 q d} \rho-1}=0
$$

We find

$$
\rho^{2}=1
$$

which implies

$$
\rho_{-}=-1, \rho_{+}=1 .
$$

For $\rho_{+}=1$, the amplitude function of fractal type, $\theta_{2}(z) \cong \cosh (q z)$, is symmetric, similar to the states of fractal type with regard to the (spatial) reflectivity against the origin. Then, the permitted values equation of the fractal energy of these states, $E_{S}$, has the following concrete form:

$$
\tan \left[k_{S}(l-d)\right]=-\frac{\operatorname{coth}\left(q_{S} d\right)}{q_{S}} k_{S},
$$


where

$$
\begin{aligned}
& k_{S}=\left[\frac{E_{S}}{2 m_{0} \lambda^{2}(d t)^{\left(\frac{4}{D_{F}}\right)-2}}\right]^{\frac{1}{2}}, \\
& q_{S}=\left[\frac{V_{0}-E_{S}}{2 m_{0} \lambda^{2}(d t)^{\left(\frac{4}{D_{F}}\right)-2}}\right]^{\frac{1}{2}} .
\end{aligned}
$$

For $\rho_{-}=-1$, the amplitude function of fractal type $\theta_{2}(z) \cong \sinh (q z)$, so the states of fractal type will be antisymmetric, and the permitted values equation, $E_{A}$, becomes

$$
\tan \left[k_{A}(l-d)\right]=-\frac{\tanh \left(q_{A} d\right)}{q_{A}} k_{A},
$$

where

$$
\begin{aligned}
& k_{A}=\left[\frac{E_{A}}{2 m_{0} \lambda^{2}(d t)^{\left(\frac{4}{D_{F}}\right)-2}}\right]^{\frac{1}{2}}, \\
& q_{A}=\left[\frac{V_{0}-E_{A}}{2 m_{0} \lambda^{2}(d t)^{\left(\frac{4}{D_{F}}\right)-2}}\right]^{\frac{1}{2}} .
\end{aligned}
$$

Now, some consequences are notable: The presence of the barrier (of finite height $V_{0}$ ) between $-d$ and $d$ leads to the splitting of the fundamental level $E_{0}$ into two sublevels, $E_{S}, E_{A}$, accounting for the two states of fractal type, symmetric and antisymmetric, respectively, in which the system can be found. In the following, the above results will be calibrated to the LPP dynamics given by our experimental data. Therefore:

(i) At a global scale resolution, it can be seen as a self-structuring of the laser ablation plasma through its separation on two oscillation modes. We distinguish a Coulomb oscillations mode, corresponding to the fast structure, and a thermal oscillation mode, corresponding to the slow structure. The identification of the plasma structures, at a certain scale resolution, can be performed by assuming that the quantity $\Delta E=\left|E_{A}-E_{S}\right|$ is small compared to the plasma potential $E_{0}$, i.e., $\Delta E<<E_{0}$, which implies the fact that $q_{A, S}$ are very close to

$$
q_{0}=\left[\frac{V_{0}-E_{0}}{2 m_{0} \lambda^{2}(d t)^{\left(\frac{4}{D_{F}}\right)-2}}\right]^{\frac{1}{2}},
$$

and also considering that

$$
\frac{\operatorname{coth}\left(q_{0} d\right)}{\tanh \left(q_{0} d\right)}=\left(\frac{e^{q_{0} d}+1}{e^{q_{0} d}-1}\right)^{2}>1,
$$

with $d>0$. This indicates that the fast structure is induced by the anti-symmetric energy state $E_{A}$, while the slower structure is induced by the symmetrical one $E_{S}$.

Potential barriers $V_{0}-E_{A}$ and $V_{0}-E_{S}$ are attributed to the double layers. In the empirical reality, the increase in the laser beam energy will lead to an increase in the intensity of the acceleration field during the Coulomb explosion [24] and, subsequently, of each of the two double layers; in the fractal geometry, this will be assimilated with the $V_{0}-E_{0}$ parameters.

(ii) Assuming the functionality of the scale resolution superposition principle [32-34], at a global scale resolution (overall plasma plume), the following fractal equations are valid:

$$
\begin{aligned}
& E_{A}=2 m_{0} \lambda(d t)^{\left(\frac{2}{D_{F}}\right)-1} f_{A}, \\
& E_{S}=2 m_{0} \lambda(d t)^{\left(\frac{2}{D_{F}}\right)-1} f_{S},
\end{aligned}
$$

where $f_{\mathrm{A}}$ is the characteristic frequency of the Coulomb oscillation modes and $f_{\mathrm{s}}$ is the characteristic frequency of the thermal oscillation modes. As $E_{A}>E_{S}$, the oscillation frequency of the Coulomb 
mode will always be higher than that of the thermal oscillation mode, as it was shown experimentally in Figure 3.

(iii) At a global scale resolution, Equations (26) and (28) with the restrictions,

$$
\begin{gathered}
k_{A}(l-d)<<1, q_{A} d \approx q_{0} d<<1, \\
k_{S}(l-d)<<1, q_{S} d \approx q_{0} d<<1, \\
l>>d,
\end{gathered}
$$

take the following approximate form:

$$
E_{A} \approx E_{S} \approx\left(V_{0}-E_{0}\right)+\frac{6 m_{0} \lambda^{2}(d t)^{\left(\frac{4}{D_{F}}\right)-2}}{l^{2}},
$$

or even a simpler form, by multiplying with $1 / 2 m_{0} \lambda(d t)^{\left(\frac{2}{D_{F}}\right)-1}$ :

$$
f_{A} \approx f_{S}=f \approx f_{0}+\frac{3 \lambda(d t)^{\left(\frac{2}{D_{F}}\right)-1}}{l^{2}},
$$

where the following notations have been used:

$$
\begin{gathered}
f=\frac{E_{A}}{2 m_{0} \lambda(d t)^{\left(\frac{2}{D_{F}}\right)-1}}=\frac{E_{S}}{2 m_{0} \lambda(d t)^{\left(\frac{2}{D_{F}}\right)-1}}, \\
f_{0}=\frac{V_{0}-E_{0}}{2 m_{0} \lambda(d t)^{\left(\frac{2}{D_{F}}\right)-1}} .
\end{gathered}
$$

This leads to the value of $f$ decreasing with the increase in $l$ and increasing with the increase in the $\left(V_{0}-E_{0}\right)$. In such a context, according to our previous considerations, the theoretical model at a local scale resolution $\lambda_{A}(d t)^{\left(\frac{2}{D_{F}}\right)-1}$ and $\lambda_{S}(d t)^{\left(\frac{2}{D_{F}}\right)-1}$ can describe the experimental behavior of the laser-produced plasmas presented in Figure 3.

(iv) In our model, the parameter $\lambda(d t)^{\left(2 / D_{F}\right)-1}$ corresponds to the scale resolution. The parameter can be defined as:

$$
\lambda(d t)^{\left(2 / D_{F}\right)-1}=\xi(d t)^{\left(2 / D_{F}\right)-1} u(d t)^{\left(2 / D_{F}\right)-1},
$$

where $\xi(d t)^{\left(2 / D_{F}\right)-1}$ is the mean free path and $u(d t)^{\left(2 / D_{F}\right)-1}$ is the expansion velocity of the laser ablation plasma. As the mean free path is inversely proportional to the collision cross-section, the collision cross-section has a functional dependence on the laser fluence. Therefore, through Equation (36), the oscillation frequency increases with the increase in the laser fluence.

(v) Considering that the energies from Equation (32) are of kinetic nature, $E_{A}=\frac{m_{0} v_{A}^{2}}{2}$ and $E_{S}=\frac{m_{0} v_{S}^{2}}{2}$, coupled with the fact that the separation of the plasma plume on the two oscillating modes can be expanded at local scale resolutions, $\lambda_{A}(d t)^{\left(\frac{2}{D_{F}}\right)-1}$ and $\lambda_{S}(d t)^{\left(\frac{2}{D_{F}}\right)-1}$, respectively, from Equation (32):

$$
f_{A}=\frac{v_{A}^{2}}{4 \lambda_{A}(d t)^{\left(\frac{2}{D_{F}}\right)-1}}, f_{S}=\frac{v_{S}^{2}}{4 \lambda_{S}(d t)^{\left(\frac{2}{D_{F}}\right)-1}},
$$

where $v_{A}$ is identified as the velocity of the fast structure and $v_{S}$ is the velocity of the slower one. Particularly, for the ablation plasma dynamics associated with the scale transitions such as correlative-non-correlative processes (the fractal dimension of the movement curves has the value $D_{F}$ $=2[31])$, Equation (37) becomes

$$
f_{A}=\frac{v_{A}^{2}}{4 \lambda_{A}}, f_{S}=\frac{v_{S}^{2}}{4 \lambda_{S}}
$$


Moreover,

$$
\lambda_{A}=\frac{k T_{e}}{m \overline{v_{A}}}, \lambda_{S}=\frac{k T_{e}}{m \overline{v_{S}}}
$$

where $k$ is the Boltzmann constant, $T_{\mathrm{e}}$ is the electron temperature, and $m$ is the atomic mass of the ions. $\overline{v_{A}}$ is the ion-electron collision frequency at Coulomb-scale resolutions, and $\overline{v_{S}}$ is the collision frequency at thermal-scale resolutions, causing Equations (38) with (39) to become

$$
f_{A}=\frac{m \overline{v_{A}} v_{A}^{2}}{k T_{e}}, f_{S}=\frac{m \overline{v_{S}} v_{S}^{2}}{k T_{e}} .
$$

Let us now perform a numerical evaluation of Equation (40) using the experimental data as input parameters. The experimental data were presented in the previous section and the simulated results are synthesized in Table 1. We notice a good correlation between the experimental data and the simulated ones. Small discrepancies can be seen for the second structure at larger distances where the experimental data are noisier and the values can be affected by errors. In addition, this representation showcases once again the versatility of the fractal theoretical approach, as once the model is calibrated onto a specific measurement, it can offer results in very good agreement with the empirical data.

Table 1. Comparison between the experimental and theoretical values of the ionic oscillating frequency for the first (a) and second (b) plasma structure.

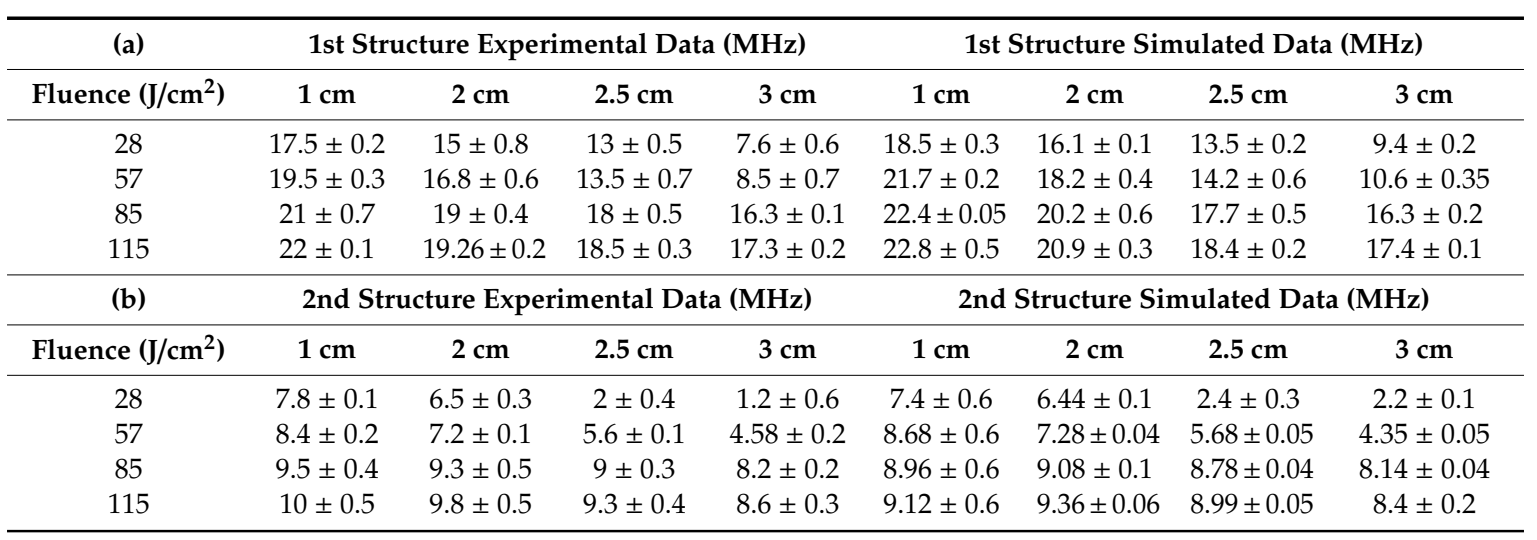

\subsection{Mutual Conditionings of the Plasma Structures through Joint Invariant Functions}

The fact that the presence of two plasma structures can be explained through the self-structuring of the plasma plume on two oscillation modes shows the mutual conditionings between these two structures. In order to characterize these conditionings, we must assume, according to the empirical proof presented in Figure 2, that the two plasma structures are characterized by two differential equations of dampened oscillator type for the charged particle densities. By using the group properties of these differential equations (invariances of SL(2R) type, algebras of Lie type, etc. [43]) and the Stoka theorem [41], we will build the joint invariant functions on the two isomorph SL(2R)-type groups. These functions will describe the mutual conditionings between the two plasma structures. Following this, let us consider the Lie algebra base associated with the SL(2R) group given by the infinitesimal generators $[32,34]$ :

$$
A_{1}=\frac{\partial}{\partial h}+\frac{\partial}{\partial \bar{h}}, A_{2}=h \frac{\partial}{\partial h}+\bar{h} \frac{\partial}{\partial \bar{h}}, A_{3}=h^{2} \frac{\partial}{\partial h}+\bar{h}^{2} \frac{\partial}{\partial \bar{h}^{2}},
$$

and its commutation relations:

$$
\left[A_{1}, A_{2}\right]=A_{1},\left[A_{2}, A_{3}\right]=A_{3},\left[A_{3}, A_{1}\right]=-2 A_{2}
$$

where $h$ is a complex amplitude and $\bar{h}$ is its complex conjugate. We assume that this group would characterize the Coulomb structure behavior. 
Let us also consider the Lie algebra base associated with the SL(2R) group given by the infinitesimal generators:

$$
B_{1}=\frac{\partial}{\partial y}+\frac{\partial}{\partial \bar{y}^{\prime}}, B_{2}=y \frac{\partial}{\partial y}+\bar{y} \frac{\partial}{\partial y}, B_{3}=y^{2} \frac{\partial}{\partial y}+\bar{y}^{2} \frac{\partial}{\partial y},
$$

and its commutation relations:

$$
\left[B_{1}, B_{2}\right]=B_{1},\left[B_{2}, B_{3}\right]=B_{3},\left[B_{3}, B_{1}\right]=-2 B_{2},
$$

where $y$ is a complex amplitude and $\bar{y}$ is its complex conjugate. We assume now that this group would characterize the thermal structure behavior.

The above-mentioned groups are isomorphic, i.e., the joint invariant functions $F$ at the actions of these groups will be obtained as solutions of Stoka's equations [43]:

$$
A_{l} F+B_{l} F=0, \quad l=1,2,3,
$$

or, in a more explicit way,

$$
\begin{aligned}
& \frac{\partial F}{\partial h}+\frac{\partial F}{\partial \bar{h}}+\frac{\partial F}{\partial y}+\frac{\partial F}{\partial \bar{y}}=0 \\
& h \frac{\partial F}{\partial h}+\bar{h} \frac{\partial F}{\partial \bar{h}}+y \frac{\partial F}{\partial y}+\bar{y} \frac{\partial F}{\partial \bar{y}}=0 \\
& h^{2} \frac{\partial F}{\partial h}+\bar{h}^{2} \frac{\partial F}{\partial \bar{h}}+y^{2} \frac{\partial F}{\partial z}+\bar{y}^{2} \frac{\partial F}{\partial \bar{y}}=0
\end{aligned}
$$

The rank's system is 3; therefore, only one independent integral exists. This is the cross-ratio generated by means of the following relation:

$$
\frac{h-y}{h-\bar{y}}: \frac{\bar{h}-y}{\bar{h}-\overline{\bar{y}}}=\sigma,
$$

where $\sigma$ is real and positive. Now, any joint invariant function here is a regular function of this cross-section. In particular, for $\sigma=\tanh \tau$ with $\tau$ arbitrary, through Equation (47), $z$ is connected to $h$ by means of the following relation:

$$
y=u+v h_{0}
$$

with

$$
\begin{aligned}
& h=u+i v \\
& h_{0}=-i \frac{\cosh \tau-e^{-i \alpha} \sinh \tau}{\cosh \tau+e^{-i \alpha} \sinh \tau} \\
& \Delta \tau=0
\end{aligned}
$$

where $\Delta$ is the Laplace operator and $\alpha$ is real. Equations (48) and (49) establish a relation between the current density amplitude of the two plasma structures. Moreover, through Equation (49), we showcase a self-modulation in amplitude of the ionic current density through the Stoler-type transformation $[44,45]$. We showcase Stoler-type transformations with an important role in the theory of coherent states, meaning on the charge ionization/neutralization processes [43,44]. The possibility to use Stoler-type transformation for the analysis of laser-produced plasma dynamics has more profound implications. The existence of coherent states implies that the individual ejected particles are coherent (through the double-layer characteristic to each laser ablation plasma structure), and there is a connecting tissue amongst the ablated particle cloud. This aspect of the laser-produced plasma was empirically showcased by our group in [10], where we show that at all laser ablation scales, there are mathematical functions connecting series of materials, a property that is inherent to a fractal model. As the particularity of a fractal model is the use of zoom in/zoom out processes, we can still find a coherence between the plasma structures (Coulomb or thermal). This is seen experimentally as, although the individual values differ for each structure, the overall trends are respected regardless of the nature of the structure. Another important role is played by the ionization/neutralization processes. The ionization processes are directly connected to the collisions during expansion, accounted for in 
the framework of our fractal model through the fractalization degree and the dependences presented in Equation (36). Our mathematical approach is, therefore, a complex one accounting for the main processes occurring during the expansion of the laser-produced plasma, and, through an adequate calibration of the model, we can offer a quantitative analysis of the oscillatory phenomena.

\section{Conclusions}

Transient plasmas generated by ns-laser ablation on an $\mathrm{Mg}$ target at high irradiation fluences were investigated by means of the Langmuir probe method and fractal analysis. The electrical investigations revealed the presence of a dual structure for the ionic and electronic saturation currents. An oscillatory behavior was observed for short evolution times and a classical quasi-exponential decrease was observed for longer times. Two oscillation frequencies were found in all irradiation conditions: A few tens of $\mathrm{MHz}$ and a few $\mathrm{MHz}$. Each frequency is associated with an individual plasma structure. Spaceand time-resolved investigations were performed, revealing an exponential decrease in the main plasma parameters during expansion coupled with an increase in the plasma potential at high measurement distances. The effect of the laser fluence on both oscillatory and classical plasma parameters was investigated. Similar empirical evolutions were found to be described by a steep increase followed by saturation at higher fluences.

The dynamics of laser-produced plasmas were described through the fractal theory of motion given by Schrödinger regimes of fractal type. The calibration of such dynamics through a fractal-type tunneling effect for physical systems with spontaneous symmetry breaking allows the self-structuring of laser-produced plasma in the two structures (Coulomb and thermal substructures) based on its separation on different oscillations modes, as well as the determination of some characteristics involved in the self-structuring process: The evolution of the structure's oscillation frequencies with the target probe distance, and with the laser fluence, the average values of these frequencies. The mutual conditionings between the two structures were given as a joint invariant function on the action of two isomorphous groups of SL(2R). Their isomorphism implies structure amplitude self-modulation through Stoler-type transformation (i.e., through charge generation/neutralization processes).

Author Contributions: Conceptualization, I.M., S.A.I. and M.A.; methodology, I.M., S.A.I. and M.A.; investigation, I.M., S.A.I. and M.A.; writing-original draft preparation, I.M., S.A.I., F.N. and M.A.; writing-review and editing, I.M., S.A.I., F.N. and M.A.; visualization, S.A.I. and I.M.; supervision, M.A. All authors have read and agreed to the published version of the manuscript.

Funding: This work was financially supported by the National Authority for Scientific Research and Innovation in the framework of the Nucleus Program -16N/2019 and by a grant of the Ministry of Research and Innovation, CNCS-UEFISCDI, project number PN-III-P4-ID-PCE-2016-0355, within PNCDI III.

Conflicts of Interest: The authors declare no conflict of interest.

\section{References}

1. Dijkkamp, D.; Venkatesan, T.; Wu, X.D.; Shaheen, S.A.; Jisrawi, N.; Min-Lee, Y.H.; McLean, W.L.; Croft, M. Preparation of $\mathrm{Y}-\mathrm{Ba}-\mathrm{Cu}$ oxide superconductor thin films using pulsed laser evaporation from high $\mathrm{T}_{\mathrm{C}}$ bulk material. Appl. Phys. Lett. 1987, 51, 619-621. [CrossRef]

2. Bulai, G.; Trandafir, V.; Irimiciuc, S.A.; Ursu, L.; Focsa, C.; Gurlui, S. Influence of rare earth addition in cobalt ferrite thin films obtained by pulsed laser deposition. Ceram. Int. 2019, 45, 20165-20171. [CrossRef]

3. Craciun, D.; Socol, G.; Stefan, N.; Dorcioman, G.; Hanna, M.; Taylor, C.R.; Lambers, E.; Craciun, V. Applied Surface Science The effect of deposition atmosphere on the chemical composition of TiN and $\mathrm{ZrN}$ thin films grown by pulsed laser deposition. Appl. Surf. Sci. 2014, 302, 124-128. [CrossRef]

4. Yang, Q.I.; Zhao, J.; Zhang, L.; Dolev, M.; Fried, A.D.; Marshall, A.F.; Risbud, S.H.; Kapitulnik, A. Pulsed laser deposition of high-quality thin films of the insulating ferromagnet EuS. Appl. Phys. Lett. 2014, 104, 082402. [CrossRef]

5. Craciun, V.; Doina, C. Reactive pulsed laser deposition of TiN. Appl. Surf. Sci. 1992, 54, 75-77. [CrossRef] 
6. Dorcioman, G.; Socol, G.; Craciun, D.; Argibay, N.; Lambers, E.; Hanna, M.; Taylor, C.R.; Craciun, V. Applied Surface Science Wear tests of $\mathrm{ZrC}$ and $\mathrm{ZrN}$ thin films grown by pulsed laser deposition. Appl. Surf. Sci. 2014, 306, 33-36. [CrossRef]

7. Amoruso, S.; Bruzzese, R.; Velotta, R.; Spinelli, M.; Vitiello, M.; Wang, X. Characterization of $\mathrm{LaMnO}_{3}$ laser ablation in oxygen by ion probe and opticalemission spectroscopy. Appl. Surf. Sci. 2005, 248, 45-49. [CrossRef]

8. Irimiciuc, S.; Bulai, G.; Agop, M.; Gurlui, S. Influence of laser-produced plasma parameters on the deposition process: In situ space- and time-resolved optical emission spectroscopy and fractal modeling approach. Appl. Phys. A 2018, 124, 1-14. [CrossRef]

9. Aragón, C.; Aguilera, J.A. Characterization of laser induced plasmas by optical emission spectroscopy: A review of experiments and methods. Spectrochim. Acta Part B 2008, 63, 893-916. [CrossRef]

10. Irimiciuc, S.A.; Nica, P.E.; Agop, M.; Focsa, C. Target properties-plasma dynamics relationship in laser ablation of metals: Common trends for fs, ps and ns irradiation regimes. Appl. Surf. Sci. 2019, 506, 144926. [CrossRef]

11. Irimiciuc, S.A.; Gurlui, S.; Bulai, G.; Nica, P.; Agop, M.; Focsa, C. Langmuir probe investigation of transient plasmas generated by femtosecond laser ablation of several metals: Influence of the target physical properties on the plume dynamics. Appl. Surf. Sci. 2017, 417, 108-118. [CrossRef]

12. Irimiciuc, S.A.; Mihaila, I.; Agop, M. Experimental and theoretical aspects of a laser produced plasma. Phys. Plasmas 2014, 21, 093509. [CrossRef]

13. Chen, J.; Lippert, T.; Ojeda-G-P, A.; Stender, D.; Schneider, C.W.; Wokaun, A. Langmuir probe measurements and mass spectrometry of plasma plumes generated by laser ablation of $\mathrm{La}_{0.4} \mathrm{Ca}_{0.6} \mathrm{MnO}_{3}$. J. Appl. Phys. 2014, $116,073303$.

14. Kumari, S.; Kushwaha, A.; Khare, A. Spatial distribution of electron temperature and ion density in laser induced ruby $\left(\mathrm{Al}_{2} \mathrm{O}_{3}: \mathrm{Cr}^{3+}\right)$ plasma using Langmuir probe. J. Instrum. 2012, 7, C05017. [CrossRef]

15. Schou, J.; Toftmann, B.; Amoruso, S. Dynamics of a laser-produced silver plume in an oxygen background gas. In High-Power Laser Ablation V; International Society for Optics and Photonics: New Mexico, NM, USA, 2004; Volume 5448, pp. 22-26.

16. Harilal, S.S.; Bindhu, C.V.; Tillack, M.S.; Najmabadi, F.; Gaeris, A.C. Internal structure and expansion dynamics of laser ablation plumes into ambient gases. J. Appl. Phys. 2003, 93, 2380-2388. [CrossRef]

17. Harilal, S.S.; Issac, R.C.; Bindhu, C.V.; Nampoori, V.P.N.; Vallabhan, C.P.G. Temporal and spatial evolution of $\mathrm{C}_{2}$ in laser induced plasma from graphite target. J. Appl. Phys. 1996, 80, 3561. [CrossRef]

18. Vivien, C.; Hermann, J.; Perrone, A.; Boulmer-Leborgne, C. A study of molecule formation during laser ablation of graphite in low-pressure ammonia. J. Phys. D 1999, 32, 518-528. [CrossRef]

19. Thestrup, B.; Toftmann, B.; Schou, J.; Doggett, B.; Lunney, J.G. Ion dynamics in laser ablation plumes from selected metals at $355 \mathrm{~nm}$. Appl. Surf. Sci. 2002, 197, 175-180. [CrossRef]

20. Harilal, S.S.; Bindhu, C.V.; Nampoor, V.P.N.; Vallabhan, C.P.G. Temporal and spatial Behavior of electron density and temperature in a laser-produced plasma from $\mathrm{YBa}_{2} \mathrm{Cu}_{3} \mathrm{O}_{7}$. Appl. Spectrosc. 1998, 52, 449-455. [CrossRef]

21. Nica, P.; Agop, M.; Gurlui, S.; Focsa, C. Oscillatory Langmuir probe ion current in laser-produced plasma expansion. EPL 2010, 89, 65001. [CrossRef]

22. Tang, E.; Xiang, S.; Yang, M.; Li, L. Sweep Langmuir Probe and Triple Probe Diagnostics for Transient Plasma Produced by Hypervelocity Impact. Plasma Sci. Technol. 2012, 14, 747-753. [CrossRef]

23. Singh, S.C.; Fallon, C.; Hayden, P.; Mujawar, M.; Yeates, P.; Costello, J.T. Ion flux enhancements and oscillations in spatially confined laser produced aluminum plasmas. Phys. Plasmas 2014, 21, 093113. [CrossRef]

24. Bulgakov, A.V.; Bulgakova, N.M. Dynamics of laser-induced plume expansion into an ambient gas during film deposition. J. Phys. D 1999, 28, 1710-1718. [CrossRef]

25. Focsa, C.; Gurlui, S.; Nica, P.; Agop, M.; Ziskind, M. Plume splitting and oscillatory behavior in transient plasmas generated by high-fluence laser ablation in vacuum. Appl. Surf. Sci. 2017, 424, 299-309. [CrossRef]

26. Irimiciuc, S.A.; Agop, M.; Nica, P.; Gurlui, S.; Mihaileanu, D.; Toma, S.; Focsa, C. Dispersive effects in laser ablation plasmas. Jpn. J. Appl. Phys. 2014, 53, 116202. [CrossRef]

27. Irimiciuc, S..; Enescu, F.; Agop, A.; Agop, M. Lorenz Type Behaviors in the Dynamics of Laser Produced Plasma. Symmetry 2019, 11, 1135. [CrossRef] 
28. Irimiciuc, S.A.; Gurlui, S.; Nica, P.; Focsa, C.; Agop, M. A compact non-differential approach for modeling laser ablation plasma dynamics. J. Appl. Phys. 2017, 12, 083301. [CrossRef]

29. Marine, W.; Bulgakova, N.M.N.M.; Patrone, L.; Ozerov, I. Electronic mechanism of ion expulsion under UV nanosecond laser excitation of silicon: Experiment and modeling. Appl. Phys. A 2004, 79, 771-774. [CrossRef]

30. Merlino, R.L. Understanding Langmuir probe current-voltage characteristics. Am. J. Phys. 2007, 75, 1078. [CrossRef]

31. Nottale, L. Scale Relativity and Fractal Space-Time: An Approach to Unifying Relativity and Quantum Mechanics; Imperial College Press: London, UK, 2011.

32. Merches, I.; Agop, M. Differentiability and Fractality in Dynamics of Physical Systems; World Scientific: Singapore, 2016.

33. Agop, M.; Paun, V.P. On the New Paradigm of Fractal Theory; Fundamental and applications, Romanian Academy Publishing House: Bucharest, Romania, 2017.

34. Agop, M.; Merches, I. Operational Procedures Describing Physical Systems; CRC Press, Taylor and Francis Group: London, UK, 2019.

35. El-Nabulsi, R.A. Some implications of position dependent mass quantum fractional Hamiltonian in Quantum mechanics. Eur. Phys. J. Plus 2019, 134, 192. [CrossRef]

36. Wójcik, D.; Białynicki-Birula, I.; Życzkowski, K. Time Evolution of Quantum Fractals. Phys. Rev. Lett. 2000, 85, 5022. [CrossRef]

37. Laskin, N. Fractals and quantum mechanics. Chaos 2000, 4, 780-790. [CrossRef]

38. Golmankhaneh, A.K.; Golmankhaneh, A.K.; Balean, D. About Schrödinger Equation on Fractals CurvesImbedding inR ${ }^{3}$. Int. J. Theor. Phys. 2015, 54, 1275-1282. [CrossRef]

39. Chuprikov, N.L.; Spiridonov, O.V. A new type of solution of the Schrödinger equationon a self-similar fractal potential. J. Phys. A 2008, 41, 409801. [CrossRef]

40. Dhillon, H.S.; Kusmartsev, F.V.; Kürten, K.E. Fractal and Chaotic Solutions of the Discrete Nonlinear Schrödinger Equation in Classical and Quantum Systems. J. Nonlinear Math. Phys. 2001, 8, 38-49. [CrossRef]

41. El-Nabulsi, R.A. Path Integral Formulation of Fractionally Perturbed Lagrangian Oscillators on Fractal. J. Stat. Phys. 2018, 172, 1617-1640. [CrossRef]

42. Mandelbrot, B. The Fractal Geometry of Nature; WH Freeman Publisher: New York, NY, USA, 1993.

43. Stoka, M.I. Integral Geometry; Romanian Academy Publishing House: Bucharest, Romania, 1967.

44. Stoler, D. Equivalence Classes of Minimum Uncertainty Packets. Phys. Rev. D 1970, 1, 3217. [CrossRef]

45. Stoler, D. Equivalence Classes of Minimum-Uncertainty Packets. II. Phys. Rev. D 1972, 4, 1925. [CrossRef] 